\title{
Study of the Effect of a Shielding Cylinder on the Torque in a Permanent-Magnet Synchronous Machine Considering Two Torque-Producing Mechanisms
}

\author{
Bert Hannon, Peter Sergeant, Member, IEEE, Luc Dupré, Member, IEEE
}

Electrical Energy Laboratory, EEMMeCS, Ghent University, Valentin Vaerwyckweg 1, 9000 Gent, Belgium

\begin{abstract}
Despite an ever-growing interest, a lot of questions related to the design and operation of high-speed permanent-magnet synchronous machines remain unanswered. One aspect of such high-speed machines that requires special attention is the effect of the shielding cylinder, a conductive sleeve that is wrapped around the magnets and which' goal is to reduce the rotor losses and/or retain the magnets. Therefore this work aims at theoretically studying the effect of the shielding cylinder on the torque production. The study is performed using a 2D analytical subdomain model that accounts for slotting and the eddy-current reaction field. The torque is divided in two components, the classical torque due to interaction between the magnets and the stator currents and the torque due to interaction with the eddy-currents in the shielding cylinder. This approach is unique and results in a better insight in the machine's physics.
\end{abstract}

\section{INTRODUCTION}

Three reasons have caused a continuous increase of the interest in high-speed Permanent-Magnet Synchronous Machines. Firstly, such machines have a very high power density. This is a major advantage in modern industry were space and weight restrictions are often very important. Secondly, their relatively small dimensions reduce the required amount of rare-earth magnets. Finally, the introduction of high-speed PMSMs enables a direct-drive configuration of applications that require high speeds, such as spindles, gas turbines, etc. This implies eliminating the losses and maintenance related to the gearbox.

Although a great number of authors has already published on high-speed electric machines [1]-[4], there are still a lot of question related to the design of such machines.

One aspect of high-speed PMSMs that still needs a lot of studying is the effect of the Shielding Cylinder (SC) on the machine's performance. This shielding cylinder is a conductive sleeve that is wrapped around the magnets. The goal of the SC can either be to keep the magnets in place at very high rotational speeds and/or to protect the magnets from overheating. The latter is due the fact that the resistance of the SC is much lower than that of the magnets. Eddy-currents, which will be induced in the SC instead of in the magnets, will therefore cause less losses.

\section{A. Goal and method}

The electromagnetic torque is one of the most important design parameters in high-speed electric machines. Indeed, as power density is a major goal, the net torque is of great importance. Moreover, from a mechanical point-of-view the torque

B. Hannon, P. Sergeant and L. Dupré are with the Electrical Energy Laboratory, Ghent University, Ghent, Belgium as well as with Flanders Make, the strategic research centre for the manufacturing industry, Belgium (e-mail: Bert.Hannon@UGent.be) ripple may be critical as well. Despite a lot of publications on torque maximization and torque ripple minimization [5]-[10], the effect of the shielding cylinder on the torque production is yet to be studied in detail.

Earlier, the authors presented a paper on torque production in PMSMs with a shielding cylinder [11]. It was shown that there are two torque-producing mechanisms in such machines. Firstly, there is the classical interaction between the permanent magnets on the rotor and the currents in the stator. The torque due to this interaction is referred to as the torque produced in the magnets $\left(T_{P M}\right)$. Secondly, a part of the torque is produced due to interaction between, on the one hand, the magnets and the stator currents and, on the other hand, the eddy currents in the shielding cylinder. This part of the torque production, which is referred to as the torque produced in the shielding cylinder $\left(T_{S C}\right)$, can be explained intuitively by regarding the $\mathrm{SC}$ as the rotor of a solid-rotor induction machine, which interacts with the asynchronous components of the magnetic field. Together, $T_{P M}$ and $T_{S C}$ make up the total torque $(T)$. These torque components were earlier used to study the effect of a variety of machine parameters such as the tooth width, the pitch factor, etc. on the torque production [11], [12]. However, the effect of the design parameters of the SC itself, i.e. its thickness $\left(t_{S C}\right)$ and its conductivity $\left(\sigma_{S C}\right)$, have not been considered yet. Therefore, this work aims at studying the effect of the shielding cylinder's design on the torque and its components thereby gaining more insight in the operation of high-speed PMSMs. The performed study is based on the 2D analytical subdomain model, presented in [13] and the torque calculation method presented in [11]. Both are briefly introduced in Section III.

\section{B. Outline}

The work is structured in 5 sections. In Section II the geometry of the studied machine is presented and its 
parameters are defined. Section III explains how the magnetic vector potential (A) can be calculated analytically, taking into account both the slotting effect and the eddy-current reaction field. Starting from the obtained A, a method to calculate the torque and its components is presented and validated in Section III as well. The actual parameter study of $t_{S C}$ and $\sigma_{S C}$ is presented in Section IV. The work is concluded in Section V.

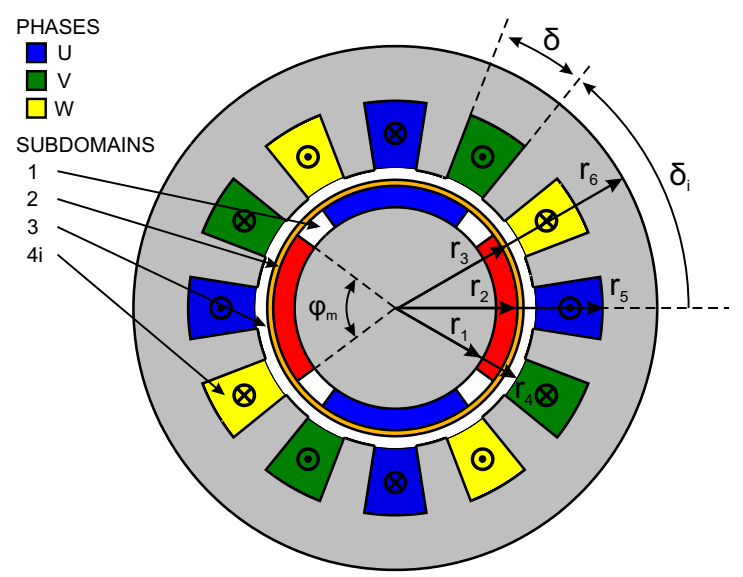

Fig. 1. Geometry and subdomains of the studied machine

\section{GeOMETRY}

The studied machine is a three-phase permanent-magnet synchronous machine with surface-mounted magnets and a $\mathrm{SC}$, as shown in Figure 1. The machine has two pole pairs and is equipped with single-layer distributed windings. The number of slots per pole and per phase is 1 . Every slot is indicated by an index $i$. When referred to the stator coordinate system, its angular position is $\delta_{i}$ :

$$
\delta_{i}=-\frac{\delta}{2}+\frac{2 \pi}{N_{s}} i \text { with } 0 \leq i \leq N_{s}-1
$$

where $N_{s}$ is the number of slots.

The applied current is sinusoidal and the material chosen for the SC is copper. An overview of the machine's parameters, and their values is listed in Table I.

\section{TORQUE CALCULATION}

As already mentioned in Section I, torque is produced in two ways in PMSMs with a SC. Firstly, the presence of permanent magnets and stator coils results in torque production $\left(T_{P M}\right)$. This part of the torque production is similar to the torque production in a classical PMSM. Secondly, asynchronous components of the magnetic field will interact with the SC as in an induction machine. This part of the torque production is called $\left(T_{S C}\right)$. As shown in the following, both $T_{P M}$ and $T_{S C}$ consist of a part that is time-independent, i.e. the net torque, and a part that is time-dependent, i.e. the torque ripple.

A brief introduction on how the torque and its components are calculated is presented in this section, a more extensive discussion can be found in [11].
TABLE I

PARAMETERS OF THE STUDIED MACHINE

\begin{tabular}{lll}
\hline Symbol & Parameter & Value \\
\hline$r_{1}$ & Rotor yoke radius & $22.5 \mathrm{~mm}$ \\
$r_{2}$ & PM radius & $25.0 \mathrm{~mm}$ \\
$r_{3}$ & SC radius & $26.5 \mathrm{~mm}$ \\
$r_{4}$ & Air gap outer radius & $28.5 \mathrm{~mm}$ \\
$r_{5}$ & Slot outer radius & $40.4 \mathrm{~mm}$ \\
$r_{6}$ & Machine outer radius & $50.0 \mathrm{~mm}$ \\
$l_{s}$ & Stack length & $200.0 \mathrm{~mm}$ \\
$N_{s}$ & Number of slots & 12 \\
$\delta$ & Slot opening angle & $\frac{\pi}{N_{s}} \mathrm{rad}$ \\
$B_{r e m}$ & Remanent induction & $1.2 \mathrm{~T}$ \\
$p$ & Number of pole pairs & 2 \\
$\varphi_{m}$ & Magnet span & $0.8 \frac{\pi}{p} \mathrm{rad}$ \\
$I$ & Current (amplitude) & $54.37 \mathrm{~A}$ \\
$f$ & Electrical frequency & $1000 \mathrm{~Hz}$ \\
$N_{t}$ & Number of turns per coil & 10 \\
$m$ & Number of phases & 3 \\
$\sigma_{S C}$ & SC conductivity & $6.94 \cdot 10^{5} \Omega \mathrm{m}$ \\
$\sigma_{P M}$ & PM conductivity & $4.83 \cdot 10^{7} \Omega \mathrm{m}$ \\
$n_{s}$ & Rated speed & $30.000 \mathrm{rpm}$ \\
$P_{n}$ & Rated power & $29 \mathrm{~kW}$ \\
\hline
\end{tabular}

\section{A. Assumptions}

To enable an analytical approach the following assumptions are adopted:

- infinite permeability of the rotor and stator iron,

- relative permeability of the SC, the PM and stator slots $\mu_{r}=1$,

- no eddy currents in the slots, the magnets or the rotor and stator iron,

- radial slot boundaries,

- no end effects.

\section{B. $2 D$ analytical subdomain model}

The torque calculation starts from the Fourier-based analytical model described in [13]. It calculates the axial component of the magnetic vector potential (A) by applying the subdomain technique. This technique entails dividing the machine in a number of regions, called subdomains. Those subdomains are referred to by an index $\nu$. As shown in Figure 1, $\nu=1$ refers to the magnet area, $\nu=2$ refers to the shielding cylinder and $\nu=3$ refers to the air gap. Finally, every slot is a separate subdomain, referred to by $\nu=4 i$ where $i$ is the slot number. The resulting model describes the magnetic vector potential as an exponential Fourier series over time and space:

$$
\begin{aligned}
& A^{(\nu)}(r, \varphi, t)= \\
& \quad \sum_{n=-\infty}^{\infty} \sum_{k=-\infty}^{\infty} A_{n, k}^{(\nu)}(r) e^{j\left(k \varphi+(k-n) \omega_{m} t+k \varphi_{0}\right)}
\end{aligned}
$$

if $\nu=1,2,3$ and

$$
\begin{aligned}
& A^{(\nu)}(r, \varphi, t)= \\
& \quad \sum_{n=-\infty}^{\infty} \sum_{k=-\infty}^{\infty} A_{n, k}^{(\nu)}(r) e^{j\left(\frac{k \pi}{\delta}\left(\varphi-\delta_{i}\right)+\left(\frac{k \pi}{\delta}-n\right) \omega_{m} t+\frac{k \pi}{\delta} \varphi_{0}\right)}
\end{aligned}
$$

if $\nu=4 i$.

In the above, $\omega_{m}$ is the machine's mechanical pulsation and $k$ and $n$ represent the time- and spatial-harmonic order 
respectively. Note that mechanical periodicities are considered. This implies that the fundamental harmonic orders in the two pole-pair machine of Figure 1 are $n= \pm 2$ and $k= \pm 2$. Note as well that every harmonic component $(n, k)$ of the magnetic field is separately available. The $r$-dependent parts of (2) and (3) are discussed in [13].

In the lower subdomains $(\nu=1 \cdots 3)$, the rotational speed of the field's $(n, k)$-component with respect to the rotor can be calculated as:

$$
\omega_{n, k}=\frac{k-n}{k} \omega_{m}
$$

\section{Torque calculation}

Based on the obtained magnetic vector potential, the torque acting on a given volume can be computed by integrating Maxwell's stress tensor over the surface of that volume. This implies that the torque acting on the entire rotor can be calculated by considering the entire rotor volume. If there are no induced currents in the rotor yoke, $T_{P M}$ can be calculated by considering the volume defined by the rotor yoke and the magnets. The torque produced in the shielding cylinder can then be calculated as the difference of $T$ and $T_{P M}$.

To compute $T$, a cylinder, coaxial to the machine's axis, with radius $r_{3}$ is chosen as integration surface. This results in:

$$
\begin{aligned}
T(t) & =\frac{l_{s} r_{3}^{2}}{\mu_{0}} \int_{0}^{2 \pi} B_{r}^{(3)}\left(r_{3}, \varphi, t\right) B_{\varphi}^{(3)}\left(r_{3}, \varphi, t\right) \mathrm{d} \varphi \\
& =2 \pi \frac{l_{s} r_{3}^{2}}{\mu_{0}} \sum_{n=-\infty}^{\infty} \sum_{s=-\infty}^{\infty} T_{n, s} e^{-j(n+s) \omega_{m} t}
\end{aligned}
$$

where

$$
T_{n, s}=\sum_{k=-\infty}^{\infty} B_{r, n, k}^{(3)}\left(r_{3}\right) B_{\varphi, s,-k}^{(3)}\left(r_{3}\right)
$$

$B_{r, n, k}(r)$ and $B_{\varphi, s,-k}(r)$ are the $r$-dependent parts of $B_{r, n, k}(r, \varphi, t)$ and $B_{\varphi, s,-k}(r, \varphi, t)$, which, in turn, are found from the definition of the magnetic vector potential.

To calculate $T_{P M}$, Maxwell's stress tensor is integrated over the surface of the cylinder defined by the rotor yoke and the magnets:

$$
T_{P M}(t)=2 \pi \frac{l_{s} r_{2}^{2}}{\mu_{0}} \sum_{n=-\infty}^{\infty} \sum_{s=-\infty}^{\infty} T_{P M, n, s} e^{-j(n+s) \omega_{m} t}
$$

where

$$
T_{P M, n, s}=\sum_{k=-\infty}^{\infty} B_{r, n, k}^{(1)}\left(r_{2}\right) B_{\varphi, s,-k}^{(1)}\left(r_{2}\right)
$$

Finally, $T_{S C}$ can be calculated by subtracting $T_{P M}$ from $T$ :

$$
T_{S C}(t)=T(t)-T_{P M}(t)
$$

The above describes a method to calculate the torque $(T)$ and its components $\left(T_{S C}\right.$ and $\left.T_{P M}\right)$ in an analytical way. The expressions for the torque (5), (7) and (9) indicate that net torque can be produced by both synchronous and asynchronous field components, as long as their time-harmonic orders are opposite.

The sign of $T_{S C, n,-n}$ depends on the relative speed of $(k, n)$ with respect to the rotor. If $(k, n)$ rotates faster than the rotor, it will result in positive torque. If $(k, n)$ rotates slower than the rotor, it will result in negative torque.

A more extensive description of the torque calculation can be found in [11].

\section{Validation}

The above described method is validated by comparing the results with those obtained from a Finite-Element Model (FEM). The applied parameters are the ones listed in Table I. The highest order of the time and spatial harmonics is chosen to be 50 .

The comparison is shown in Figure 2, it can be seen that there is a very good agreement between the analytical model and the finite-element model.

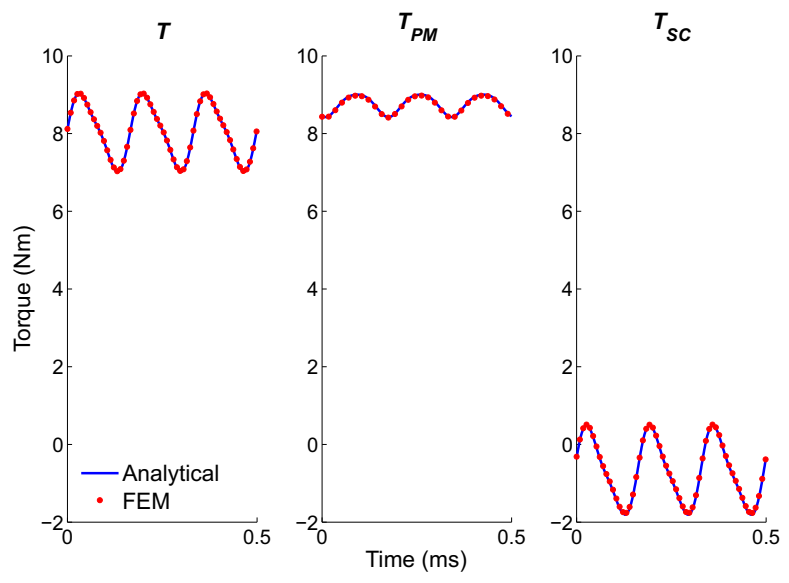

Fig. 2. Comparison of the torque obtained with the analytical model and the finite-element model

Figure 2 shows that $T_{S C \text {, net }}$ is negative. This is because, due to its distributed windings and sinusoidal stator current, the eddy currents in this machine are mainly due to relative movement of the magnets with respect to the slots. From Lenz' law, it can easily be understood that those eddy currents will result in a negative torque. By means of illustration, Figure 3 shows a harmonic map of the studied machine's magnetic field. It is clear that the dominant asynchronous field components rotate undersynchronously, i.e. at a speed that is lower than the synchronous speed of the rotor.

$T_{S C, \text { net }}$ may become positive if the oversynchronous harmonic combinations become more important than the undersynchronous ones. Figure 3 illustrates that this may be the case if the current contains higher time-harmonic orders and the slotting effect is reduced, e.g. by adding teeth tips.

Note that $T_{S C \text {,net }}$ being negative suggests that adding a SC to the studied machine may not be interesting from a torquedensity point-of-view. This suspicion will indeed be confirmed in the following.

Moreover, for this particular machine the SC does not reduce the overall eddy-current losses in the rotor. Indeed, using Joule's law and the Poynting theorem, the eddy-current losses in the magnets and the shielding cylinder can be calculated. If the machine described in Figure 1 and Table $\mathrm{I}$ is equipped with an AISI 303 stainless steel SC $\left(\sigma_{S C}=1.38 .10^{6} \frac{S}{m}\right)$ to retain the magnets, the eddy-current losses in the magnets and 
the SC are $206 \mathrm{~W}$ and $858 \mathrm{~W}$ respectively, amounting to a total of $1064 \mathrm{~W}$ of eddy-current losses. If the SC of the same machine were to be replaced by air, the eddy-current losses in the rotor equal those in the magnets; $209 \mathrm{~W}$. Adding a SC thus quintuples the rotor's eddy-currents losses.

It is therefore clear that adding a SC to this machine might not improve its electromagnetic performance. However, as the goal of this work is to identify trends and to get a better understanding of high-speed PMSMs, rather than to design an actual machine, this is not an issue.

Note that whether or not a SC improves the electromagnetic properties strongly depends on the machine's geometry and its stator-current density. Zhou et al. have, for example successfully used a SC to reduce the rotor loss of a high-speed PMSM [14].

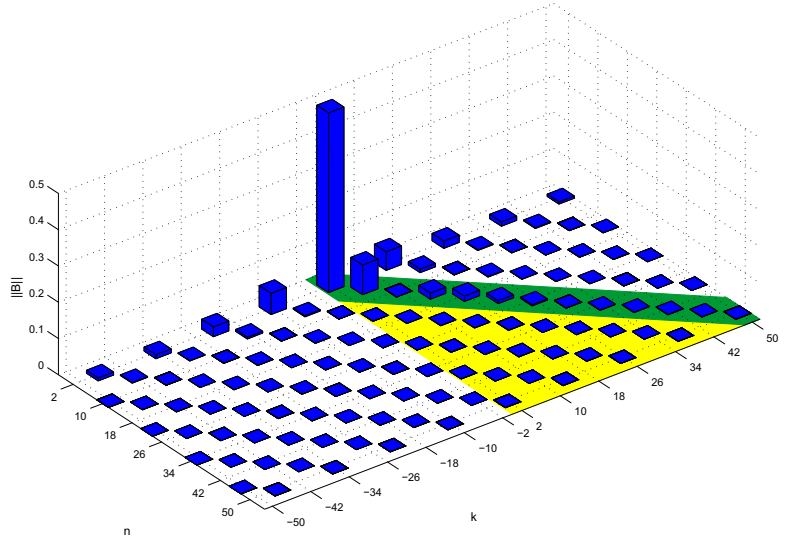

Fig. 3. Harmonic map of the validated machine: green, yellow and white indicate synchronous, oversynchronous and undersynchronous harmonic combinations

\section{PARAMETER STUdy}

As already mentioned in the introduction, this work provides a theoretical study of the effect of the shielding cylinder's thickness $\left(t_{S C}\right)$ and conductivity $\left(\sigma_{S C}\right)$ on the machine's torque. Two aspects of the torque, and its components, are regarded; the net torque and the torque ripple. The net torque values are calculated as the average of the torque over one mechanical period, they are indicated as $T_{n e t}, T_{P M, n e t}$ and $T_{S C, \text { net }}$. In this work the torque ripple is expressed as a percentage of $T_{\text {net }}$ :

$$
\left\{\begin{array}{l}
\Delta T_{\%}=100 \% \cdot \frac{\max (T)-\min (T)}{T_{n e t}} \\
\Delta T_{P M, \%}=100 \% \cdot \frac{\max \left(T_{P M}\right)-\min \left(T_{P M}\right)}{T_{n e t}} \\
\Delta T_{S C, \%}=100 \% \cdot \frac{\max \left(T_{S C}\right)-\min \left(T_{S C}\right)}{T_{n e t}}
\end{array}\right.
$$

\section{A. SC thickness}

In a first study, the effect of the shielding cylinder's thickness $\left(t_{S C}=r_{3}-r_{2}\right)$ on the torque is regarded. This is done by varying $r_{3}$ while $r_{2}$ is kept constant. To ensure a comparison that is as fair as possible, all the other distances are kept constant. This implies that $r_{1}$ is constant while the variation of $r_{4}, r_{5}$ and $r_{6}$ is equal to the variation of $r_{2}$. None of the other parameters, listed in Table I, are changed.

The expectation is that the net torque will decrease when the thickness of the shielding cylinder is increased. Indeed, the net torque level is proportional to the average magnetic flux density, which, in turn, is inversely proportional to the thickness of the effective air gap $\left(t_{A G, e f f}\right)$. Obviously, $t_{A G, \text { eff } f}$, which is determined by all the non-magnetic material between the stator and rotor iron, is affected when changing $t_{S C}$. The above would imply that the evolution of $T_{n e t}$ as a function of $t_{S C}$ can be predicted when the torque at a reference thickness is known:

$$
T_{n e t}\left(t_{S C}\right)=T_{n e t}\left(t_{S C, r e f}\right) \frac{t_{A G, \text { ef } f, r e f}\left(t_{S C, r e f}\right)}{t_{A G, \text { eff }}\left(t_{S C}\right)}
$$

Note that $t_{A G, \text { eff }}$ is not simply proportional to $t_{S C}$. The slotting effect has to be taken into account. This can be done using Carter's factor $\left(k_{c}\right)$ :

$$
k_{c}=\frac{1}{1-\tau_{c} \frac{N_{s} \delta}{2 \pi}}
$$

with:

$$
\tau_{c}=\frac{2}{\pi}\left(\arctan \frac{w_{s}}{2 t_{A G}}-\frac{2 t_{A G}}{w_{s}} \ln \sqrt{1+\left(\frac{w_{s}}{2 t_{A G}}\right)^{2}}\right)
$$

where $t_{A G}$ is the combined thickness of the magnets, the SC and the air gap $\left(t_{A G}=r_{4}-r_{1}\right)$ and $w_{s}$ is the width of the slots at the border with the air gap $\left(w_{s}=\delta r_{4}\right)$. The thickness of the effective air gap can now be calculated as:

$$
t_{A G, e f f}=k_{c} t_{A G}
$$

The expectation is thus that the evolution of $T_{n e t}\left(t_{S C}\right)$ may be predicted using (11), where $t_{A G, \text { eff }}$ is calculated as in (14). In Figure 4 Carter's torque prediction, where the torque at $t_{S C}=0$ was chosen as a reference, is depicted with a black line, the analytically calculated torque is indicated with blue dots.

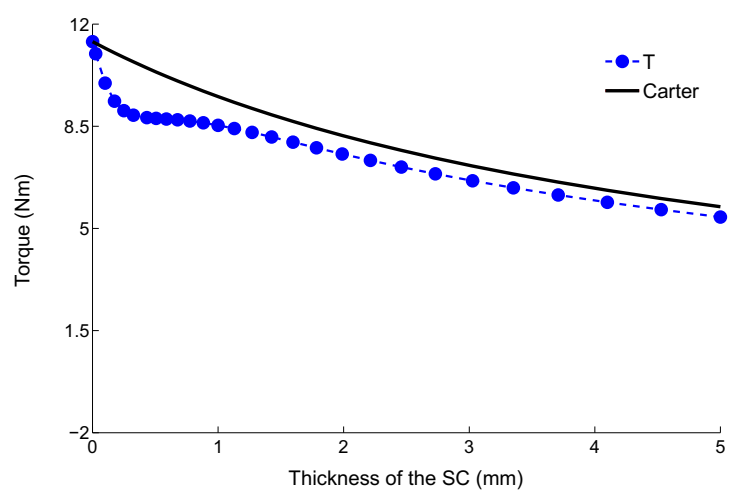

Fig. 4. Comparison of the actual net torque level and its prediction according to Carter

It can be seen that there is a large deviation between Carter's torque prediction and the actual torque. The reason is that the prediction is not taking into account the torque production in the shielding cylinder. This can easily be validated by analyzing the torque components, as shown in Figure 5. An 
important observation is that (11) is now successfully used to predict $T_{P M, n e t}$ while $T_{S C \text {,net }}$ accounts for the difference between $T_{n e t}$ and $T_{P M, n e t}$.

Two effects determine the evolution of $T_{S C, \text { net }}\left(t_{S C}\right)$. An increasing $t_{S C}$ results in a decrease of the SC's resistance, which in turn leads to higher eddy currents and thus a larger importance of $T_{S C, \text { net }}$. On the other hand $T_{S C, \text { net }}$ also suffers from a larger effective air gap, which implies a decreasing importance of $T_{S C, n e t}$ at increasing $t_{S C}$. Initially, the increasing importance of $T_{S C, \text { net }}$ due to lower resistance dominates its decrease due to a larger effective air gap. At a given $t_{S C}$ the roles are reversed, the effect of an increasing effective air gap thickness will become dominant. The amplitude and the position of the resulting maximum depend on numerous parameters such as the geometry, the electrical frequency and the number of pole pairs.

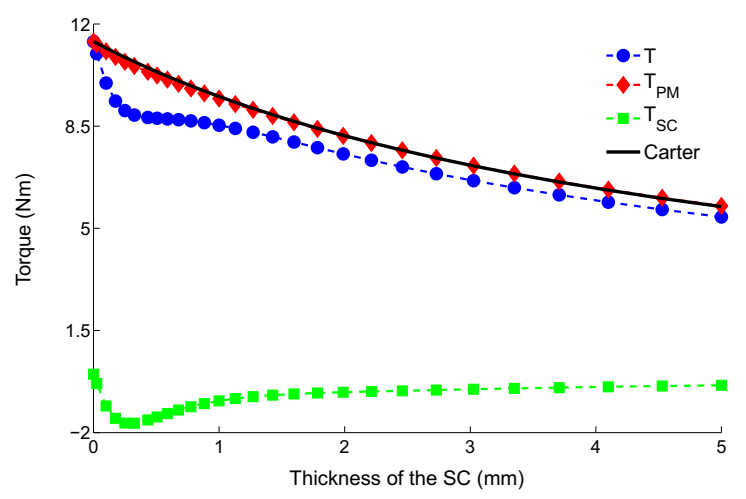

Fig. 5. Evolution of the net torque and its components in function of $t_{S C}$

In Figure 6, the ratio of the torque ripple with respect to $T_{n e t}$ is shown for the overall torque and its components. Initially, there is a minor increase of $\Delta T_{P M, \%}$. This is because $T_{n e t}$ decreases faster than $T_{P M}$ 's ripple. After this small increase, the effect of the SC is very clearly illustrated; the amount of ripple in $T_{P M}$ strongly decreases because of a higher shielding effect and a larger effective air gap. The effect of the SC stagnates when $t_{S C}$ becomes larger than the penetration depth of the dominant asynchronous harmonic combinations $\left(d_{d o m}\right)$. Which, as can be seen from Figure 3 , are $( \pm 2, \mp 10)$ and $( \pm 2, \pm 14)$. As those combinations are already greatly damped when $d_{d o m}=t_{S C}$, a further increase of $t_{S C}$ will only have a limited effect.

The penetration depth of the magnetic field's $(n, k)$ component in the SC can be approximated as:

$$
d_{n, k}=\sqrt{\frac{2}{|k-n| \omega_{m} \mu_{0} \sigma_{S C}}}
$$

The evolution of $\Delta T_{S C, \%}\left(t_{S C}\right)$ is similar to the evolution of $T_{S C, n e t}\left(t_{S C}\right)$. However, the thickness related to its maximum may be different, depending on the evolution of $T_{n e t}\left(t_{S C}\right)$. Finally, it can also be noted that the sum of $\Delta T_{P M, \%}$ and $\Delta T_{S C, \%}$ doesn't necessarily equal $\Delta T_{\%}$. This indicates a time shift between $T_{P M}(t)$ and $T_{S C}(t)$. This time shift is illustrated in Figure 7, where the torque and its components are plotted over one electrical period if $t_{S C}=1 \mathrm{~mm}$. The reason for $\Delta t$ is the different nature of $T_{P M}$ and $T_{S C}$. $T_{P M}$ will reach a

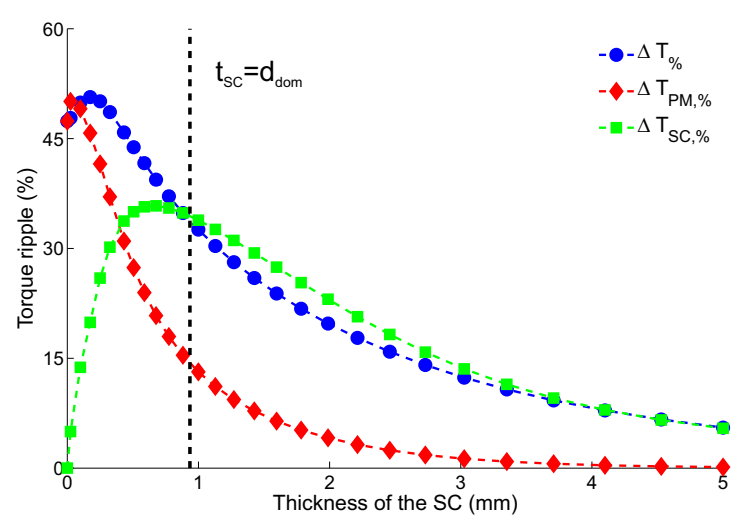

Fig. 6. Evolution of the torque and its components in function of $t_{S C}$

maximum depending on the alignment of the magnets and the stator teeth, while $T_{S C}$ reaches a maximum depending on the rate at which the magnetic field changes. These effects do not necessarily occur at the same moment.

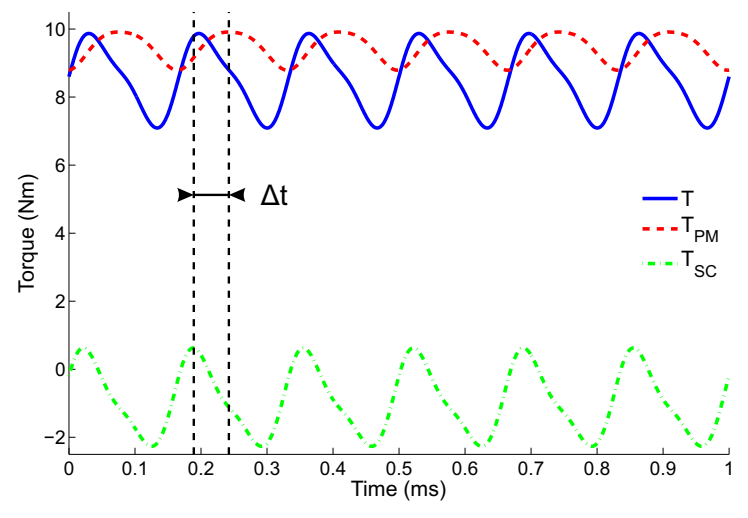

Fig. 7. The torque and its components as a function of time if $t_{S C}=1 \mathrm{~mm}$

Studying the torque at various thicknesses of the shielding cylinder has illustrated that considering the torque components helps to get a better understanding of the machine's physics. For the net torque level for example, it was shown that $T_{P M}$ evolves as expected from a classical synchronous machine. This is not true for $T_{S C}$, which explains why the evolution of $T$ does not meet the initial expectations.

\section{B. SC conductivity}

In contrast to the previous section, the parameter study in this section maintains a constant $t_{S C}$ of $1.5 \mathrm{~mm}$. The parameter that is varied is the shielding cylinder's conductivity $\left(\sigma_{S C}\right)$. The range in which $\sigma_{S C}$ is regarded is $\left[0,10^{8}\right]$. Note that such extreme conductivities are not realistic. That is not an issue since the goal here is to identify trends rather than to model a real machine.

The net torque as a function of $\sigma_{S C}$ is given in Figure 8 .

Figure 8 shows only very little variation of $T_{P M, n e t}$, as $T_{P M, n e t}$ is mainly caused by synchronous harmonic combinations, this was expected. However, Figure 9, which plots $T_{P M, n e t}$ separately, does show an initial decrease, followed by a slight increase after which there is a steady decrease. The reason for these, relatively small, variations is that, in contrast 


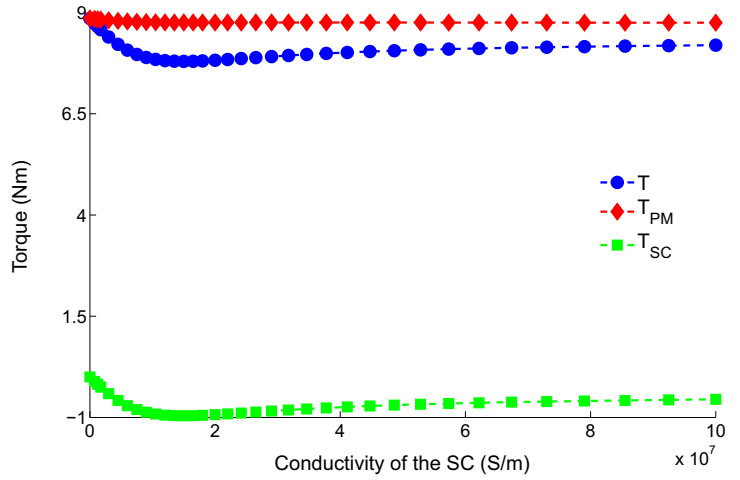

Fig. 8. The torque and its components as a function of $\sigma_{S C}$

to what might have been expected, the variation of asynchronous harmonic combinations do affect the synchronous harmonic combinations. Indeed, through the slotting effect, all spatial harmonic orders are related.

By studying the harmonic content of Maxwell's stress tensor and the magnetic field, an explanation for the evolution of $T_{P M, n e t}\left(\sigma_{S C}\right)$ can be found. Initially, a decrease of $B_{\phi, \pm 2, \pm 2}^{(1)}$ dominates the evolution of $T_{P M, n e t}$. As $( \pm 2, \pm 2)$ are the fundamental harmonic combinations, i.e. the stator current has a time-harmonic order of \pm 2 , they contribute to a positive $T_{P M, n e t}$. A decrease of those harmonic combinations will thus result in a decreasing $T_{P M, n e t}$. This is in line with Figure 9. After this initial decrease, the decrease of $B_{\phi, \pm 6, \pm 6}^{(1)}$ will shortly be dominant. As there are no stator currents with $n= \pm 6$, harmonic combinations $( \pm 6, \pm 6)$ produce a negative $T_{P M, n e t}$. Their decrease therefore results in a small increase of $T_{P M, n e t}$. Finally, the decrease of harmonic combinations $( \pm 2, \pm 2)$ dominates again, further reducing $T_{P M, n e t}$.

Note that the strongest variation in $T_{P M, n e t}$ is observed prior to the $t_{S C}=d_{d o m}$ line. This supports the claim that the variation of the asynchronous harmonic combinations propagates to the synchronous combinations through the slotting effect, thereby affecting $T_{P M, n e t}$.

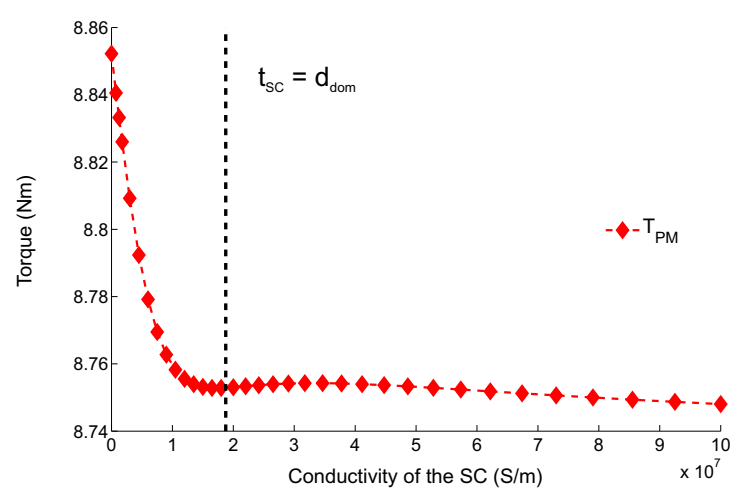

Fig. 9. $T_{P M, n e t}$ as a function of $\sigma_{S C}$

The evolution of $T_{S C, n e t}\left(\sigma_{S C}\right)$ is plotted in Figure 10 . Initially an almost linear increase of its absolute value is noted. However, after reaching a maximum $\left|T_{S C \text {, net }}\right|$, becomes inversely proportional to $\sqrt{\sigma_{S C}}$. This evolution is very similar to the speed-torque characteristic of an induction machine
[15] and can, qualitatively, be explained in a similar way. Indeed, as mentioned in Section I, the shielding cylinder may be regarded as the rotor of a solid-rotor induction machine. This means that, for a given component of the magnetic field $(n, k)$, the torque production in the SC depends on the stator resistance $\left(R_{s}\right)$, the resistance of the $\mathrm{SC}\left(R_{S C}\right)$, the stator leakage inductance $\left(L_{l s}\right)$, the rotor leakage inductance $\left(L_{l r}\right)$, the magnetization inductance $\left(L_{m}\right)$, the slip $\left(s_{n, k}\right)$ and the pulsation $\left(\omega_{n, k}\right)[15]$ :

$$
\begin{aligned}
& T_{S C, n e t, n, k} \sim \\
& \left(R_{s}+\left(1+\frac{L_{l s}}{L_{m}}\right) \frac{R_{S C}}{s_{n, k}}\right)^{2}+\omega_{n, k}^{2}\left(L_{l s}+\left(1+\frac{L_{l s}}{L_{m}}\right) L_{l r}\right)^{2}
\end{aligned}
$$

Following Pouillet's law (17), $R_{S C}$ is inversely proportional to $\sigma_{S C}$.

$$
R_{S C}=\frac{l_{s}}{\sigma_{S C} S_{S C}}
$$

with $l_{s}$ the stack length and $S_{S C}$ the surface of the SC.

However, this is only true for low $\sigma_{S C}$. At high conductivities, the skin effect is no longer negligible. The conductive surface of the SC is then not constant, but inversely proportional to $\sqrt{\sigma_{S C}}$. According to Pouillet's law $R_{S C}$ will then be inversely proportional to $\sqrt{\sigma_{S C}}$. The threshold value, after which $R_{S C}$ may no longer be assumed inversely proportional to $\sigma_{S C}$, is the conductivity at which $d_{d o m}$ equals the thickness of the SC. At low conductivities of the shielding cylinder $R_{S C}$ will thus be high and will dominate the denominator of (16). Indeed:

$$
\begin{aligned}
& R_{S C} \frac{1+\frac{L_{l s}}{L_{m}}}{s_{n, k}} \gg R_{s} \\
& R_{S C} \frac{1+\frac{L_{l s}}{L_{m}}}{s_{n, k}} \gg \omega_{n, k}\left(L_{l s}+\left(1+\frac{L_{l s}}{L_{m}}\right) L_{l r}\right)
\end{aligned}
$$

Equation (16) can then be approximated as:

$$
\begin{aligned}
T_{S C, \text { net }, n, k} & \sim \frac{R_{S C}}{\left(\left(1+\frac{L_{l s}}{L_{m}}\right) \frac{R_{S C}}{s_{n, k}}\right)^{2}} \\
& \sim \frac{1}{R_{S C}} \\
& \sim \begin{cases}\sigma_{S C} & \text { if } d_{d o m}>t_{S C} \\
\sqrt{\sigma_{S C}} & \text { if } d_{d o m}<t_{S C}\end{cases}
\end{aligned}
$$

Taking into account that, in this study, the point at which $d_{d o m}=t_{S C}$ is situated after the area in which $\left|T_{S C, n e t}\right|$ increases, the above confirms the linear behavior at low $\sigma_{S C}$ in Figure 10.

At high $\sigma_{S C}, R_{S C}$ will be low and thus:

$$
R_{S C} \frac{1+\frac{L_{l s}}{L_{m}}}{s_{n, k}} \ll R_{s}
$$


Which implies that (16) can be approximated as:

$$
\begin{aligned}
T_{S C, n e t, n, k} & \sim \frac{R_{S C}}{R_{s}^{2}+\omega_{n, k}^{2}\left(L_{l s}+\left(1+\frac{L_{l s}}{L_{m}}\right) L_{l r}\right)^{2}} \\
& \sim R_{S C} \\
& \sim \begin{cases}\frac{1}{\sigma_{S C}} & \text { if } d_{d o m}>t_{S C} \\
\frac{1}{\sqrt{\sigma_{S C}}} & \text { if } d_{d o m}<t_{S C}\end{cases}
\end{aligned}
$$

This shows that, at high $\sigma_{S C}, T_{S C \text {, net }}$ will decrease proportionally to $\frac{1}{\sigma_{S C}}$ until $d_{d o m}=t_{S C}$ after which $\left|T_{S C, \text { net }}\right|$ becomes proportional to $\frac{1}{\sqrt{\sigma_{S C}}}$. This is also confirmed by Figure 10, where it should be noted that the conductivity for which $d_{d o m}=t_{S C}$ is located in the transition area between an increasing and a decreasing $T_{S C, n e t}$. This implies that, in this parameter study, only the decrease proportional to $\frac{1}{\sqrt{\sigma S C}}$ is visible. A final remark is that the maximum of $\left|T_{S C, \text { net }}\right|\left(\sigma_{S C}\right)$ depends on the ratio between the resistances and the inductances and thus on the entire machine geometry.

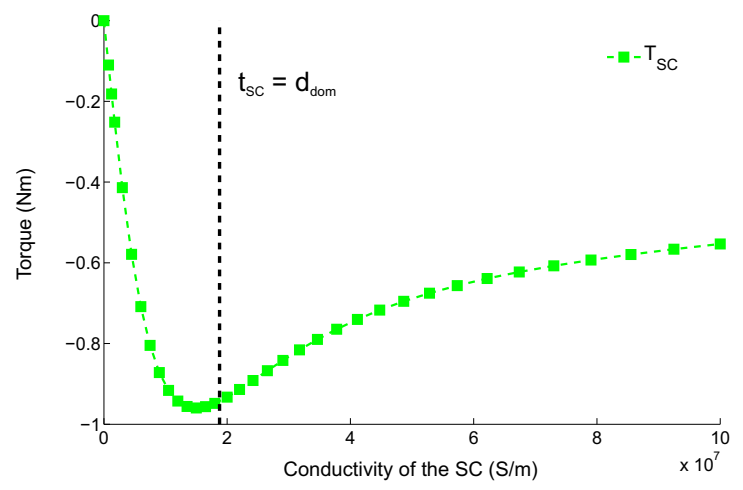

Fig. 10. $T_{S C, \text { net }}$ as a function of $\sigma_{S C}$

In Figure 11, the percentage torque ripple as a function of $\sigma_{S C}$ is shown.

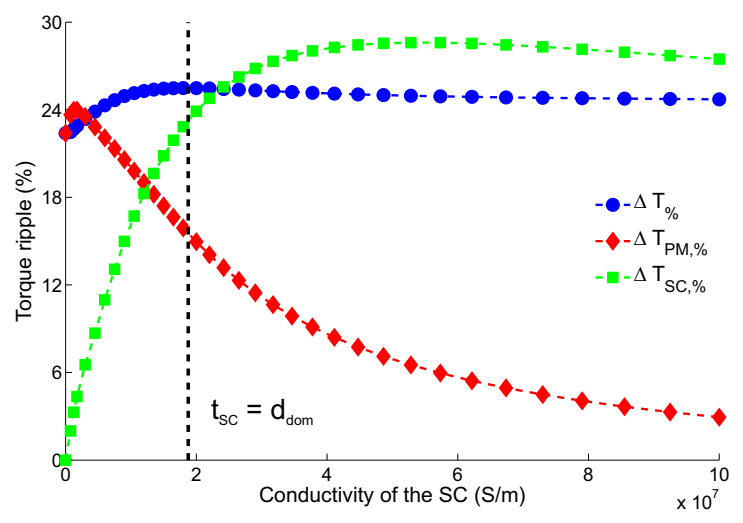

Fig. 11. $\Delta T_{\%}$ and its components as a function of $\sigma_{S C}$

It can be seen that there is a shift of the ripple from $T_{P M}$ to $T_{S C}$ when $\sigma_{S C}$ is increased. This is explained by a stronger shielding effect. Note that the small increase at very low $\sigma_{S C}$ is again explained by a stronger decrease of $T_{n e t}$ than of
$T_{P M}$ 's ripple.

The total torque ripple is almost constant. The small variation of $\Delta T_{\%}$ is due to the variation of $T_{n e t}\left(\sigma_{S C}\right)$. This is confirmed by Figure 12, which shows an evolution that is inverse to the evolution of $T_{n e t}\left(\sigma_{S C}\right)$.

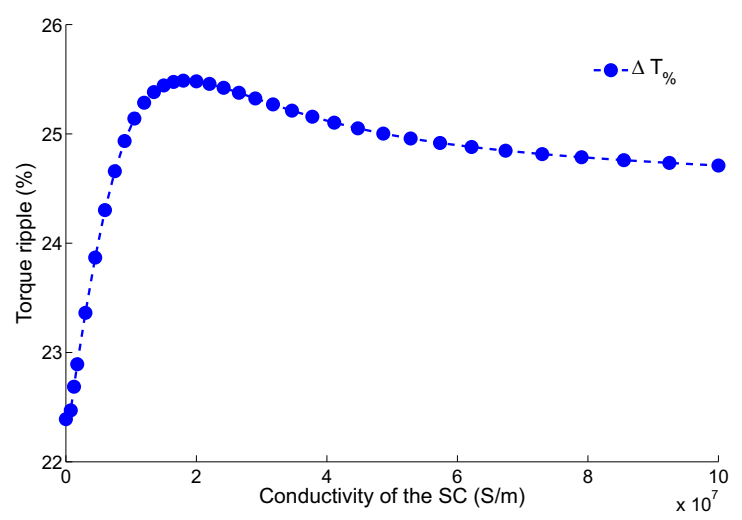

Fig. 12. $\Delta T_{\%}$ as a function of $\sigma_{S C}$

$\Delta T_{P M, \%}\left(\sigma_{S C}\right)$ shows an evolution that is similar to the evolution of $T_{P M, n e t}\left(\sigma_{S C}\right)$, but its maximum is shifted towards a lower value of $\sigma_{S C}$. The latter is due to the initial increase of $T_{n e t}\left(\sigma_{S C}\right)$.

Overall, the evolution of $\Delta T_{S C, \%}\left(\sigma_{S C}\right)$ is as expected. The increase of $\sigma_{S C}$ implies a better shielding effect which, in turn, translates in an increasing torque ripple. The increase stagnates when $d_{d o m}$ becomes smaller than $t_{S C}$, because at that moment the $\mathrm{SC}$ already blocks most of the asynchronous content.

This parameter study again highlighted the benefit of dividing the torque in its components. Studying the net torque as a function of $\sigma_{S C}$ proved that $T_{S C}$ indeed has the same characteristics as the torque in an induction machine. It also showed that, unlike the expectation, $T_{P M}$ does exhibit a dependency of $\sigma_{S C}$. Although this dependency might be considered negligible, it did lead to concluding that the presence of a SC affects the magnetic field's synchronous components. Study of the torque ripple learned that, although there is a shift of torque ripple from the magnets to the $\mathrm{SC}$, the total torque ripple is almost constant.

\section{CONCLUSION}

The goal of this work was to identify physical processes in PMSMs that are equipped with a SC. This was done by theoretically studying the effect of the shielding cylinder's thickness and conductivity on the torque and its components. The latter being the torque produced in the magnets and the torque produced in the shielding cylinder, as defined in Section I. The performed studies are based on an analytical model that calculates the magnetic vector potential [13] and a method to calculate the torque and its components [11].

The parameter study, presented in Section IV, indeed proved that a better understanding of the machine's physics can be obtained using the above described approach. The study of $t_{S C}$, for example, showed why there is a deviation of Carter's torque prediction in high-speed PMSM's. Studying the effect 
of $\sigma_{S C}$ on the torque production showed that the torque production in the shielding cylinder is indeed similar to the torque production in induction machines. Another interesting finding from the parameter studies is that, unlike expected, synchronous harmonic combinations are affected by the SC.

Note that the above results show that adding a shielding cylinder to the studied machine decreases its torque density; the highest torque levels are achieved without SC. It was however discussed in Section III-D that this finding cannot be generalized. Indeed, if the stator current contains higher time-harmonic orders and the slotting effect is decreased, the SC may contribute positively to the total torque.

Although this work provides a good method to study highspeed PMSMs and an interesting insight in the machine's physics, a lot of aspects of such machines still have to be studied. It was for example shown in Section III-D that adding a SC can increase the rotor's eddy-current losses. This is potentially disastrous for the machine. Although there is already some research available [14], the effect of the SC ion the rotor losses may be an interesting topic for future research.

\section{REFERENCES}

[1] N. Bianchi, S. Bolognani, and F. Luise, "Potentials and limits of high speed PM motors," in Industry Applications Conference, 2003. 38th IAS Annual Meeting. Conference Record of the, vol. 3, Oct. 2003, pp. 1918 1925 vol.3.

[2] L. Zheng, T. X. Wu, D. Acharya, K. Sundaram, J. Vaidya, L. Zhao, L. Zhou, C. Ham, N. Arakere, J. Kapat, and L. Chow, "Design of a superhigh-speed cryogenic permanent magnet synchronous motor," IEEE Trans. Magn., vol. 41, no. 10, pp. 3823-3825, Oct. 2005.

[3] M. Shah and S.-B. Lee, "Rapid analytical optimization of eddy-current shield thickness for associated loss minimization in electrical machines," IEEE Trans. Ind. Appl., vol. 42, no. 3, pp. 642-649, May 2006.

[4] P.-D. Pfister and Y. Perriard, "Very-high-speed slotless permanentmagnet motors: Analytical modeling, optimization, design, and torque measurement methods," IEEE Trans. Ind. Electron., vol. 57, no. 1, pp. 296-303, Jan. 2010.

[5] C. A. Borghi, D. Casadei, A. Cristofolini, M. Fabbri, and G. Serra, "Application of a multiobjective minimization technique for reducing the torque ripple in permanent-magnet motors," IEEE Trans. Magn., vol. 35 , no. 5 , pp. 4238-4246, Sep. 1999.

[6] N. Bianchi and S. Bolognani, "Design techniques for reducing the cogging torque in surface-mounted PM motors," IEEE Transactions on Industry Applications, vol. 38, no. 5, pp. 1259-1265, Sep. 2002.

[7] Z. Zhu, S. Ruangsinchaiwanich, N. Schofield, and D. Howe, "Reduction of cogging torque in interior-magnet brushless machines," in Magnetics Conference, 2003. INTERMAG 2003. IEEE International, Mar. 2003, pp. ER-04.

[8] J. Güemes, A. Iraolagoitia, J. Del Hoyo, and P. Fernandez, "Torque analysis in permanent-magnet synchronous motors: A comparative study," IEEE Trans. Energy Convers., vol. 26, no. 1, pp. 55-63, Mar. 2011.

[9] S.-M. Hwang, J.-B. Eom, Y.-H. Jung, D.-W. Lee, and B.-S. Kang, "Various design techniques to reduce cogging torque by controlling energy variation in permanent magnet motors," IEEE Trans. Magn., vol. 37, no. 4, pp. 2806-2809, Jul. 2001.

[10] K.-C. Kim, D.-H. Koo, J.-P. Hong, and J. Lee, "A study on the characteristics due to pole-arc to pole-pitch ratio and saliency to improve torque performance of IPMSM," IEEE Trans. Magn., vol. 43, no. 6, pp. 2516-2518, Jun. 2007.

[11] B. Hannon, P. Sergeant, and L. Dupr, "Torque and torque components in high-speed permanent-magnet synchronous machines with a shielding cylinder," Mathematics and Computers in Simulation, vol. 130, pp. 7080, Dec. 2016.

[12] B. Hannon, P. Sergeant, and L. Dupré, "2D analytical torque study of slotted high-speed pmsms considering pole pairs, slots per pole per phase and coil throw," in Proceedings of XXIth International Conference on Electrical Machines, Berlin, Germany, 2014, pp. 1-7.

[13] B. Hannon, P. Sergeant, and L. Dupré, "2D analytical subdomain model of a slotted PMSM with shielding cylinder," IEEE Trans. Magn., vol. 50, 2014.
[14] F. Zhou, J. Shen, W. Fei, and R. Lin, "Study of Retaining Sleeve and Conductive Shield and Their Influence on Rotor Loss in High-Speed PM BLDC Motors," IEEE Transactions on Magnetics, vol. 42, no. 10, pp. 3398-3400, Oct. 2006.

[15] I. Boldea and S. Nasar, Electric drives, second ed., CRC Press, Boca Raton, 2006. 